\title{
COVID-19: enfoque básico para entender sus tratamientos
}

Carla Cedillo-Alvarez, ${ }^{1}$ Itzell A. Gallardo-Ortiz, ${ }^{2}$ Luis T. López, ${ }^{3}$ Sergio Montes ${ }^{3}$ y Nayeli Páez-Martínez ${ }^{*}$ 'Secretaría de la Defensa Nacional, Escuela Militar de Medicina, Sección de Investigación, Ciudad de México; ${ }^{2}$ Universidad Nacional Autónoma de México, Facultad de Estudios Superiores Iztacala, Unidad de Investigación en Biomedicina, Estado de México; ${ }^{3}$ Instituto Nacional de Neurología y Neurocirugía, Departamento de Neuroquímica, Ciudad de México; ${ }^{4}$ Instituto Politécnico Nacional, Escuela Superior de Medicina, Sección de Posgrado e Investigación, Ciudad de México. México

\section{Resumen}

EI virus SARS-CoV-2 ha sido identificado como el agente patológico causante de la pandemia de COVID-19. Aun cuando no se cuenta con un tratamiento estándar, se han probado antivirales como remdesivir y otros fármacos como cloroquina e ivermectina, que interfieren con la replicación del virus. También se han intentado algunas estrategias encaminadas a disminuir los mecanismos inmunitarios, como el uso de tocilizumab y antioxidantes naturales. Los fármacos relacionados con el sistema renina-angiotensina han resultado controversiales. Aún se debe estudiar con detalle los mecanismos de patogenicidad, así como los tratamientos controlados para proponer alguna opción terapéutica viable que evite la entrada y replicación del virus o que aumente los sistemas inmunitarios del huésped.

PALABRAS CLAVE: COVID-19. Tratamientos farmacológicos. Sistema renina-angiotensina.

\section{COVID-19: a basic approach to understanding potential treatments}

\section{Abstract}

SARS-CoV-2 virus has been identified as the causative agent of the COVID-19 pandemic. Even when no standard treatment is available, antivirals such as remdesivir and other drugs such as chloroquine and ivermectin, which interfere with viral replication, have been assayed. Some strategies aimed to reduce immune mechanisms, such as the use of tocilizumab and natural antioxidants, have also been tested. The use of drugs related to the renin-angiotensin system has been controversial. Pathogenicity mechanisms, as well as controlled treatments, still have to be studied in detail in order to propose a viable therapeutic option that prevents the entry and replication of the virus or enhances the host immune system.

KEY WORDS: Covid-19. Pharmacological treatments. Renin-angiotensin system.

\section{Posibles intervenciones terapéuticas para la infección por SARS-CoV-2}

En la lucha por contrarrestar la enfermedad emergente de COVID-19 se ha utilizado una gran variedad de fármacos; sin embargo, aún no se cuenta un tratamiento estándar.
Las propuestas de tratamiento abordan diferentes aspectos:

- Entrada del virus a las células.

- Replicación del virus.

- Reforzamiento de la inmunidad y prevención de la inflamación exacerbada.

- Control de las complicaciones.
Gac Med Mex. 2020;156:580-585

Disponible en PubMed www.gacetamedicademexico.com CC BY-NC-ND (http://creativecommons.org/licenses/by-nc-nd/4.0/). 
Se han realizado numerosos ensayos en busca de tratamientos o combinación de fármacos para lograr la recuperación o aminorar el progreso hacia las formas graves de la enfermedad. En esta revisión se describen algunas de las propuestas más importantes.

\section{Remdesivir}

El remdesivir es un análogo de la adenosina, por lo que disminuye la producción de ARN viral. ${ }^{1}$ Su uso para el tratamiento de COVID-19 aún no se encuentra aprobado, aunque continúan desarrollándose diversos estudios experimentales. En estudios in vitro se observó efectividad del remdesivir contra SARSCoV-2. En animales de experimentación, remdesivir redujo la hemorragia pulmonar y el número de copias de ARN viral. Estudios preliminares en humanos sugieren que el $70 \%$ de los pacientes tratados con este antiviral lograron mejoría, incluyendo aquellos con ventilación asistida. En un análisis multicéntrico no controlado se empleó remdesivir, $200 \mathrm{mg}$ el primer día y posteriormente $100 \mathrm{mg} /$ día durante nueve días; de 53 pacientes que recibieron el fármaco, 36 mostraron mejoría clínica $(68 \%)$. $^{2}$ En otro estudio multicéntrico aleatorizado doble ciego controlado con placebo se incluyeron 237 pacientes; el uso del remdesivir no se asoció a mejoría clínica en comparación con el grupo control. Además, se reportaron más efectos adversos en el grupo que recibió el fármaco. ${ }^{3}$ Los resultados de estas investigaciones sugieren que se requieren protocolos con mayor número de pacientes a fin de evaluar los beneficios que podría ofrecer el fármaco en cuanto al tiempo en la mejoría clínica.

\section{Rivabirina, lopinavir y ritonavir}

La ribavirina es un nucleósido sintético de la guanosina que interfiere con la síntesis del ARNm. Por su parte, el lopinavir es un inhibidor de proteasas, que al coadministrarse con una dosis baja del ritonavir mejora significativamente sus propiedades farmacocinéticas y potencia su actividad contra la proteasa de $\mathrm{VIH}-1.4,5$

En un estudio realizado en 75 pacientes con síndrome respiratorio agudo grave, se observó una reducción en las tasas de mortalidad $(2.3 \%)$ e intubación $(0 \%)$ en los pacientes inicialmente tratados con 400/100 mg de lopinavir/ritonavir cada 12 horas por 14 días. $^{6}$ En otra investigación de 199 pacientes con SARS-CoV-2, a 99 pacientes se les administró lopinavir/ritonavir y terapia estándar y fueron comparados con un grupo control que recibió solo terapia estándar; los resultados no mostraron diferencia entre grupos en el tiempo de mejoría de los pacientes.?

En otro análisis de pacientes con COVID-19 se administró 400/100 mg de lopinavir/ritonavir cada 12 horas a un grupo, mientras que otro recibió adicionalmente $400 \mathrm{mg}$ de ribavirina cada 12 horas y tres dosis de $8 \times 10^{6} \mathrm{UI}$ de interferón en días alternados. Los resultados indicaron que la triple terapia antiviral fue segura y superior a la monoterapia con lopinavir/ ritonavir en lo que respecta a la duración de la enfermedad y días de estancia hospitalaria. ${ }^{8}$ Actualmente se están llevando a cabo estudios para determinar la eficacia de diferentes dosis de rivavirina, en monoterapia o en combinación con otros fármacos, en el manejo de COVID-19. ${ }^{9}$

\section{Azitromicina}

Azitromicina es un antibiótico macrólido cuyo mecanismo de acción se basa en su unión reversible a la subunidad 50 s del ribosoma bacteriano, impidiendo la translocación. ${ }^{10}$ Este fármaco también ha mostrado propiedades antiinflamatorias e inmunomodulatorias, lo cual ha llevado al interés por su uso en infecciones virales. Los reportes muestran disminución del número de neutrófilos y de la concentración de las interleucinas (IL) $8,6,1 \beta$, del factor de necrosis tumoral $\alpha$, proteína catiónica eosinofílica y metaloproteinasa $9 .{ }^{11}$ Estos datos sugieren que la azitromicina podría disminuir la cascada de citocinas inflamatorias característica de COVID-19. La azitromicina también crea un medio poco propicio para la replicación viral. ${ }^{12} \mathrm{La}$ administración de azitromicina se inicia con $500 \mathrm{mg}$ vía oral el primer día, seguidos de $250 \mathrm{mg} /$ día durante dos a cinco días, según el criterio médico y enfermedades preexistentes del paciente. ${ }^{13}$ Otros autores evaluaron las cargas virales en los pacientes con COVID-19 tras recibir hidroxicloroquina y azitromicina; concluyeron que esta combinación de fármacos produce eliminación viral con mayor eficacia. ${ }^{14}$ Actualmente, el Instituto Nacional de Alergias y Enfermedades Infecciosas de Estados Unidos se encuentra patrocinando un estudio clínico para evaluar este fármaco en terapia combinada con hidroxicloroquina. ${ }^{15}$

\section{Cloroquina e hidroxicloroquina}

Se considera que estos fármacos son capaces de cambiar el pH de los lisosomas y, por lo tanto, de modificar la actividad de las proteasas e interferir con el ingreso del 
virus a las células. La cloroquina y la hidroxicloroquina también se caracterizan por disminuir las interleucinas proinflamatorias, en particular IL-6, por lo que su acción también podría ser benéfica en la "tormenta de citocinas". ${ }^{16}$ Además, se observó que ambos fármacos en concentraciones bajas son capaces de inhibir la replicación del virus y reducir la carga viral, en estudios in vitro y en humanos, respectivamente. Sin embargo, como desventaja se identificó que el uso de la cloroquina e hidroxicloroquina provoca efectos cardiotóxicos, efecto muy importante que es necesario considerar. ${ }^{17}$

\section{Ivermectina}

Estudios in vitro de células Vero/hSLAM infectadas con SARS-CoV-2 y tratadas con ivermectina en una concentración de $5 \mu \mathrm{M}$ mostraron una reducción casi total del ARN viral; además, no se observó toxicidad de la ivermectina en la concentración evaluada. ${ }^{18}$

El mecanismo de acción de la ivermectina aún se desconoce, sin embargo, se piensa que inhibe la importina $\alpha / \beta 1$ y proteínas nucleares del virus y del hospedero. ${ }^{12}$ La dosis varía según algunos criterios, pudiendo ser de $12 \mathrm{mg}$ en dosis única por vía oral o $12 \mathrm{mg}$ vía oral durante dos días. Varios países están empleando la ivermectina en los tratamientos de primera elección para combatir la enfermedad, tal es el caso de República Dominicana, Bolivia y Perú. Sin embargo, se considera apropiado realizar más estudios para evaluar a fondo su efectividad como antiviral. ${ }^{19}$

\section{Tocilizumab}

Este fármaco es un anticuerpo monoclonal humanizado antagonista del receptor de IL-6..$^{20,21}$ Actualmente se están llevando a cabo numerosos estudios clínicos para evaluar el uso del tocilizumab en el tratamiento de la infección por SARS-CoV-2.22 Una investigación realizada en China con 21 pacientes reportó que la adición de 4 a $8 \mathrm{mg} / \mathrm{kg}$ de tocilizumab a la terapia estándar produjo mejoría clínica, las opacidades en patrón de vidrio esmerilado en las tomografías computarizadas disminuyeron después de la administración del fármaco, 15 de 20 pacientes requirieron menos oxígeno suplementario y un paciente no lo requirió. ${ }^{23}$ Sin embargo, este estudio no fue controlado y la cantidad de pacientes analizados no es suficiente para formular mayores inferencias, por lo que se requieren más investigaciones para evaluar la eficacia de tocilizumab.

\section{Vitamina C}

La vitamina C posee capacidad antioxidante mediante la donación de un electrón al radical lipídico, lo cual detiene la reacción autoperpetuada de la peroxidación lipídica; posteriormente, la vitamina $\mathrm{C}$ se transforma en dos moléculas, el radical ascorbato y el dehidroascorbato; este último se transforma nuevamente en ascorbato por la acción de una oxidorreductasa ${ }^{24} \mathrm{La}$ dosis diaria de vitamina $\mathrm{C}$ recomendada es de 90 y $75 \mathrm{mg} /$ día en varones y mujeres sin requerimientos especiales. Se sabe que las infecciones virales generan grandes cantidades de radicales libres de oxígeno; la vitamina $\mathrm{C}$ se utiliza para neutralizar ese estrés oxidativo. ${ }^{25,26}$ Además, la vitamina C reduce la inflamación mediante la estimulación de la producción de citocinas antiinflamatorias e interferón, por lo que se le atribuye un efecto antiviral directo, ${ }^{27-29}$ de ahí que se considere su probable utilidad terapéutica en COVID-19. ${ }^{30}$

Recientemente se realizó un estudio clínico aleatorizado controlado: CITRIS-ALI, en pacientes hospitalizados a quienes se administró una dosis alta de vitamina C por infusión ( $50 \mathrm{mg} / \mathrm{kg}$ cada seis horas por 96 horas). Si bien no se identificaron diferencias significativas en la puntuación de la escala de falla orgánica secuencial (SOFA) ni mejoría en los biomarcadores de inflamación o daño vascular (proteína $C$ reactiva y trombomodulina, respectivamente), se encontró que los pacientes tratados con vitamina $\mathrm{C}$ requirieron menos días de terapia intensiva $y$, en general, de hospitalización. ${ }^{31,32}$ Las dosis altas de vitamina $\mathrm{C}$ se comportan como prooxidantes, por lo que en algunos estudios se sugieren que pequeñas dosis pueden tener mayor beneficio. ${ }^{33}$ En COVID-19, el papel de la vitamina C como tratamiento coadyuvante es aún incierto.

\section{Vitamina $D$}

La vitamina $D$ es fotosintetizada en la piel a partir del 7-dehidrocolesterol. ${ }^{34}$ Además de otras funciones, la forma activa de la vitamina $D$ (calcitriol) desempeña un papel como inmunomodulador, incrementando la respuesta inmunitaria innata e inhibiendo la respuesta inmunitaria adaptativa. ${ }^{35}$ En un estudio multicéntrico retrospectivo de 212 casos se encontraron niveles bajos de vitamina $D$ en pacientes con COVID-19 en estado crítico, reportándose una asociación con el desenlace clínico. ${ }^{36}$ La mayor causa de deficiencia de vitamina $D$ es la exposición inadecuada a la luz solar, ${ }^{37,38}$ lo que se 


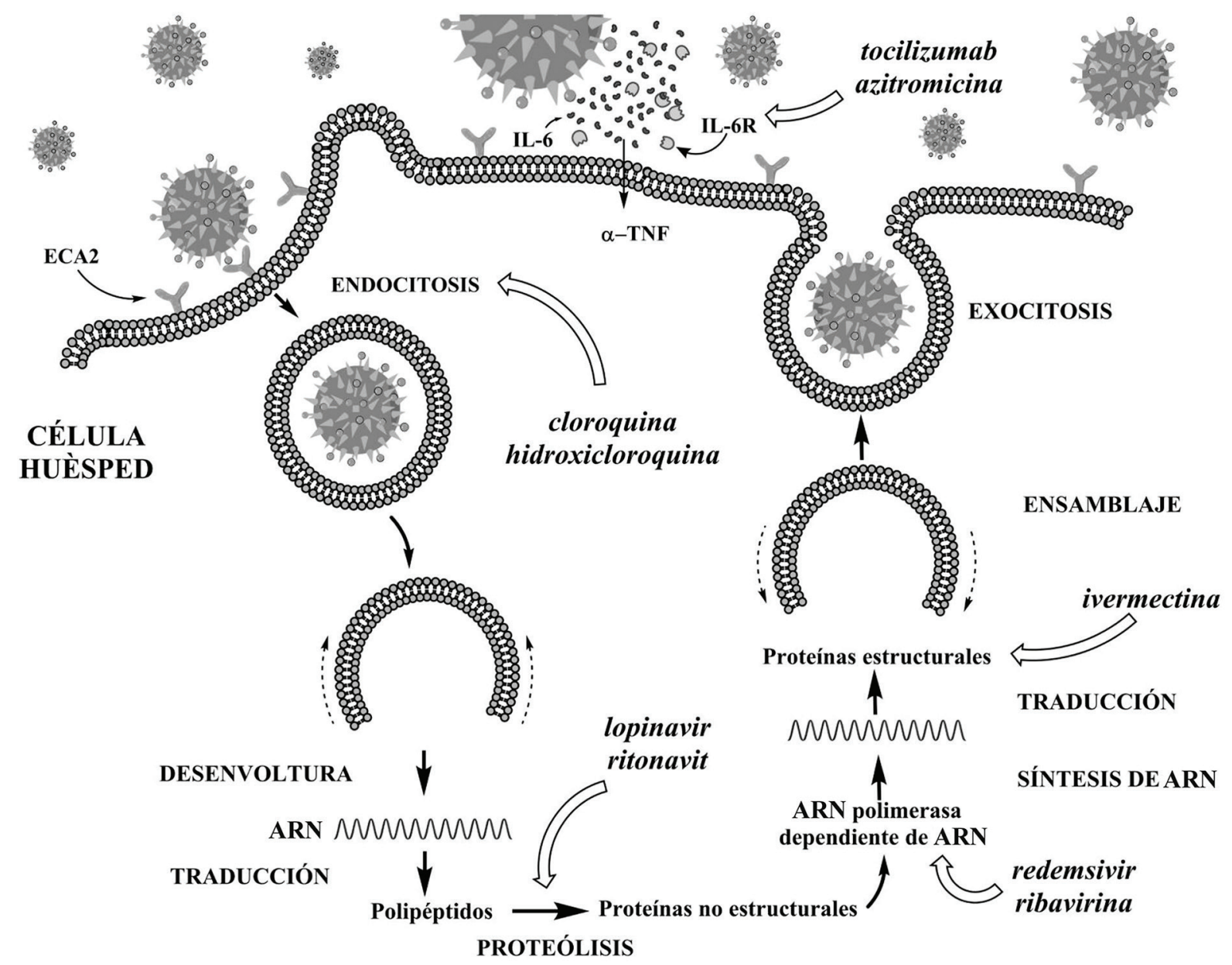

Figura 1. Tratamientos propuestos para COVID-19. Se muestran los sitios de acción probables de algunos fármacos utilizados en forma experimental y clínica contra SARS-CoV-2. Cloroquina e hidroxicloroquina inhiben la interiorización viral y la endocitosis; además, tienen efecto inmunomodulador en el huésped. Azitromicina y tocilizumab inhiben la activación de los receptores de IL-6 y sus vías de señalización, disminuyendo la quimioatracción de macrófagos y neutrófilos. Lopinavir y ritonavir inhiben la formación de proteínas no estructurales mediante el proceso de replicación viral en la proteasa similar a la 3-quimotripsina. Remdesivir y ribavirina inhiben la síntesis de ARN polimerasa dependiente de $A R N(R d R p)$. En estudios experimentales, ivermectina inhibe la síntesis de proteínas estructurales necesarias para el ensamblaje de la cápside viral.

ha sugerido como una causa de la severidad de las respuestas a la infección y la mortalidad debida a SARSCoV-2 en Europa, junto con la ingesta inadecuada de la vitamina ${ }^{39,40}$ Estos datos sugieren que la suplementación con vitamina $\mathrm{D}$ puede formar parte de la intervención terapéutica y profiláctica en COVID-19.41

\section{Tratamientos relacionados con el sistema renina-angiotensina}

La participación de los fármacos que inhiben el sistema renina-angiotensina, tales como los inhibidores de la enzima convertidora de angiotensina (IECA) y los antagonistas de los receptores de angiotensina II (ARA) son objeto de controversia en el contexto de la pandemia de COVID-19. Por un lado, existe información que muestra que la continuidad del tratamiento con IECA o ARA disminuye la morbilidad y mortalidad en pacientes con COVID-19. ${ }^{42}$ En una revisión sistemática que incluyó 12 artículos con más de 19000 casos de COVID-19 se reportó que la exposición a IECA o ARA no estuvo asociada a mayor riesgo de infección por COVID-19 ni a mayor riesgo de una infección grave o mortalidad; por el contrario, la exposición a IECA o ARA se asoció a menor riesgo de mortalidad al comparar los resultados con los obtenidos en pacientes medicados con otros antihipertensivos. $^{43}$ Mehta et al. concluyeron que no existe una asociación significativa entre el uso de IECA o ARA y la positividad de la prueba de COVID-19. Todos 
estos datos en conjunto respaldan la noción de no interrumpir los IECA o ARA en el contexto de la pandemia de COVID-19. ${ }^{44}$ Además, actualmente están en curso varios protocolos para evaluar el efecto de la administración de dichos antagonistas para el tratamiento de pacientes con COVID-19.42

En contraposición, estudios recientes de cohortes de Wuhan, China, sugieren que los peores resultados parecen ser más frecuentes en pacientes con hipertensión y diabetes, posiblemente debido a la sobreexpresión de la enzima convertidora de angiotensina tipo 2 (ECA2) en las células epiteliales alveolares debido al tratamiento con IECA o ARA. En investigaciones con animales, ARA y IECA aumentaron la expresión de ECA2, sitio de acceso de SARS-CoV-2, por lo surge la inquietud de si estos fármacos aumentan la morbilidad y mortalidad por COVID-19. ${ }^{45}$ Es importante señalar que la búsqueda minuciosa de los datos en la literatura no apoya esta asociación. ${ }^{46}$

ECA2 soluble, no asociada con la membrana, es enzimáticamente activa e inhibe parcialmente la entrada del virus hacia las células blanco. Recientemente en Europa se reportó un estudio clínico en fase II, en el que se está probando la ECA2 humana soluble de grado clínico para bloquear la infección por SARS-CoV-2. ${ }^{42}$ De hecho, se han descrito algunas variaciones estructurales de la ECA2 humana, la cual se caracteriza por su baja afinidad a la proteína viral en espiga. Los datos anteriores abren un potencial de implicaciones protectoras de la ECA2 sobre la entrada del virus ${ }^{47}$ (Figura 1).

\section{Conclusiones}

La infección por el coronavirus SARS-CoV-2 se extendió por todo el mundo en muy pocos meses. A la fecha de publicación de este manuscrito aún no se contaba con un fármaco recomendado mundialmente para tratar la enfermedad. Sin duda, los avances científicos han llevado a proponer varios medicamentos y vacunas para combatir al virus. La transmisión del SARS-CoV-2 en humanos trae consigo una reflexión sobre nuestra convivencia con la fauna silvestre, nuestros hábitos alimenticios y el cuidado de nuestra salud en general.

\section{Agradecimientos}

Agradecemos profundamente a la doctora Ethel Rubinstein Malamud, por su invaluable apoyo para la realización de este manuscrito.

\section{Conflicto de intereses}

Los autores declaran no tener conflicto de intereses alguno.

\section{Financiamiento}

El trabajo se realizó con el apoyo de los proyectos SIP-IPN, del Instituto Nacional de Psiquiatría "Dr. Ramón de la Fuente Muñiz" e Instituto Nacional de Neurología y Neurocirugía "Dr. Manuel Velasco Suárez", y de los proyectos DGAPA, PAPIIT IN226819 de la Universidad Nacional Autónoma de México.

\section{Responsabilidades éticas}

Protección de personas y animales. Los autores declaran que para esta investigación no se realizaron experimentos en seres humanos ni en animales.

Confidencialidad de los datos. Los autores declaran que en este artículo no aparecen datos de pacientes.

Derecho a la privacidad y consentimiento informado. Los autores declaran que en este artículo no aparecen datos de pacientes.

\section{Bibliografía}

1. DrugBank [sitio web]. Canadá: Remdesivir; 2019 [Actualizado 2020 Jan 14]. [Consultado 2020 May 31]. Disponible en: https://www.drugbank.ca/ drugs/DB14761\#reference-A191379

2. Grein J, Ohmagari N, Shin D, Diaz G, Asperges E, Castagna A, et al. Compassionate use of remdesivir for patients with severe Covid-19. N Engl J Med. 2020;382:2327-2336.

3. Wang Y, Zhang D, Du G, Du R, Zhao J, Jin Y, et al. Remdesivir in adults with severe COVID-19: a randomised, double-blind, placebo-controlled, multicentre trial. Lancet. 2020;395:1569-1578.

4. DrugBank [sitio web]. Canadá: Ribavirin; 2005 [Actualizado 2020 Jan 14]. [Consultado 2020 May 31]. Disponible en: https://go.drugbank.com/ drugs/DB00811

5. Cvetkovic RS, Goa KL. Lopinavir/ritonavir: a review of its use in the management of HIV infection. Drugs. 2003;63:769-802.

6. Chan KS, Lai ST, Chu CM, Tsui E, Tam CY, Wong MM, et al. Treatment of severe acute respiratory syndrome with lopinavir/ritonavir: a multicentre retrospective matched cohort study. Hong Kong Med J. 2003;9:399-406.

7. Cao B, Wang Y, Wen D, Liu W, Wang J, Fan G, et al. A trial of lopinavir-ritonavir in adults hospitalized with severe Covid-19. N Engl J Med. 2020;382:1787-1799.

8. Hung IF, Lung KC, Tso EY, Liu R, Chung TW, Chu MY, et al. Triple combination of interferon beta-1b, lopinavir-ritonavir, and ribavirin in the treatment of patients admitted to hospital with COVID-19: an open-label, randomised, phase 2 trial. Lancet. 2020;395:1695-1704.

9. Khalili JS, Zhu H, Mak NSA, Yan Y, Zhu Y. Novel coronavirus treatment with ribavirin: groundwork for an evaluation concerning COVID-19. J Med Virol. 2020;92:740-746.

10. Lorenzo-Fernández $P$, Moreno-González A, Leza-Cerro JC, Moro-Sánchez MA, Lizasoain-Hernández I. Antibióticos macrólidos y otros antibióticos. En: Velázquez, editor. Manual de farmacología básica y clínica. México: Editorial Panamericana; 2018.

11. Zimmermann P, Ziesenitz VC, Curtis N, Ritz N. The immunomodulatory effects of macrolides-a systematic review of the underlying mechanisms. Front Immunol. 2018;9:302.

12. Choudhary R, Sharma AK. Potential use of hydroxychloroquine, ivermectin and azithromycin drugs in fighting COVID-19: trends, scope and relevance. New Microbes New Infect. 2020;35:100684. 
13. Valentin F. Azithromycin and COVID-19 history and review. 2020.

14. Gautret P, Lagier JC, Parola P, Hoang VT, Meddeb L, Mailhe M, et al. Hydroxychloroquine and azithromycin as a treatment of COVID-19: results of an open-label non-randomized clinical trial. Int $\mathrm{J}$ Antimicrob Agents. 2020;56:105949.

15. National Institutes of Health [sitio web]. EE. UU.: NIH begins clinical trial of hydroxychloroquine and azithromycin to treat COVID-19; 2020.

16. Wu R, Wang L, Kuo HD, Shannar A, Peter R, Chou PJ, et al. An update on current therapeutic drugs treating COVID-19. Curr Pharmacol Rep. 2020;1-15.

17. Sanders JM, Monogue ML, Jodlowski TZ, Cutrell JB. Pharmacologic treatments for coronavirus disease 2019 (COVID-19): a review. JAMA. 2020;323:1824-1836

18. Caly L, Druce JD, Catton MG, Jans DA, Wagstaff KM. The FDA-approved drug ivermectin inhibits the replication of SARS-CoV-2 in vitro. Antiviral Res. 2020;178:104787.

19. Sharun K, Dhama K, Patel SK, Pathak M, Tiwari R, Singh BR, et al. Ivermectin, a new candidate therapeutic against SARS-CoV-2/CO VID-19. Ann Clin Microbiol Antimicrob. 2020:19:23.

20. DrugBank [sitio web]. Canadá: Tocilizumab; 2008. [Actualizado 2020 Ene 14]. [Consultado 2020 May 07]. Disponible en: https://go.drugbank.com/ drugs/DB06273

21. Food and Drug Administration [sitio web]. EE. UU.: FDA approved drug products: actemra tocilizumab intravenous or subcutaneous injection; 2013.

22. BMJ Best Practice [sitio web]. Coronavirus disease 2019 (COVID-19). Emerging treatments. BMJ Best Practice; 2020. Disponible en: https:// bestpractice.bmj.com/topics/en-us/3000168/emergingtxs

23. Xu X, Han M, Li T, Sun W, Wang D, Fu B, et al. Effective treatment of severe COVID-19 patients with tocilizumab. Proc Natl Acad Sci U S A. 2020;117:10970-10975

24. Nimse SB, Pal D. Free radicals, natural antioxidants, and their reaction mechanisms. RSC Advances. 2015:5:27986-8006.

25. Schencking M, Vollbracht C, Weiss G, Lebert J, Biller A, Goyvaerts B, et al. Intravenous vitamin $C$ in the treatment of shingles: results of a multicenter prospective cohort study. Medical Sci Monit. 2012;18:CR215CR224.

26. Mikirova N, Hunninghake R. Effect of high dose vitamin C on Epstein-Barr viral infection. Medical Sci Monit. 2014;20:725-732.

27. Boretti A, Banik BK. Intravenous vitamin C for reduction of cytokines storm in acute respiratory distress syndrome. PharmaNutrition. 2020;12:100190.

28. Colunga-Biancatelli RML, Berrill M, Marik PE. The antiviral properties of vitamin C. Expert Rev Anti Infect Ther. 2020;18:99-101.

29. Kim MS, Kim DJ, Na CH, Shin BS. A study of intravenous administration of vitamin $C$ in the treatment of acute herpetic pain and postherpetic neuralgia. Ann Dermatol. 2016;28:677-683.

30. Cheng RZ. Can early and high intravenous dose of vitamin $C$ prevent and treat coronavirus disease 2019 (COVID-19)? Med Drug Discov. $2020 ; 5: 100028$
31. Hemilä H, Chalker E. Vitamin $\mathrm{C}$ can shorten the length of stay in the ICU: a meta-analysis. Nutrients. 2019;11:708

32. Fowler AA, Truwit JD, Hite RD, Morris PE, DeWilde C, Priday A, et al. Effect of vitamin $\mathrm{C}$ infusion on organ failure and biomarkers of inflammation and vascular injury in patients with sepsis and severe acute respiratory failure: the CITRIS-ALI randomized clinical trial. JAMA. 2019;322:1261-1270.

33. Hunt C, Chakravorty NK, Annan G, Habibzadeh N, Schorah CJ. The clinical effects of vitamin $C$ supplementation in elderly hospitalised patients with acute respiratory infections. Int J Vitam Nutr Res. 1994;64:212-219.

34. Ross AC, Taylor CL, Yaktine AL, del Valle HB. Dietary reference intakes for calcium and vitamin D. EE. UU.: National Academies Press; 2011.

35. Lagishetty V, Misharin AV, Liu NQ, Lisse TS, Chun RF, Ouyang Y, et al. Vitamin $D$ deficiency in mice impairs colonic antibacterial activity and predisposes to colitis. Endocrinology. 2010;151:2423-2432.

36. Alipio M. Vitamin D supplementation could possibly improve clinical outcomes of patients infected with coronavirus-2019 (COVID-19). SSRN Electronic J. 2020

37. Holick MF, Siris ES, Binkley N, Beard MK, Khan A, Katzer JT, et al. Prevalence of vitamin D inadequacy among postmenopausal North American women receiving osteoporosis therapy. J Clin Endocrinol Metab. 2005;90:3215-3224

38. Moan J, Porojnicu AC, Dahlback A, Setlow RB. Addressing the health benefits and risks, involving vitamin $D$ or skin cancer, of increased sun exposure. Proc Natl Acad Sci U S A. 2008;105:668-673.

39. Spiro A, Buttriss JL. Vitamin D: an overview of vitamin D status and intake in Europe. Nutr Bull. 2014:39:322-350.

40. Laird E, Rhodes J, Kenny RA. Vitamin D and Inflammation: potential implications for severity of Covid-19. Ir Med J. 2020;113:81-87.

41. Jakovac H. COVID-19 and vitamin D-is there a link and an opportunity for intervention? Am J Physiol Endocrinol Metab. 2020;318:E589.

42. Saavedra JM. COVID-19, angiotensin receptor blockers, and the brain. Cell Mol Neurobiol. 2020:40:667-674.

43. Zhang $X, Y u$ J, Pan LY, Jiang HY. ACEI/ARB use and risk of infection or severity or mortality of COVID-19: a systematic review and meta-analysis. Pharmacol Res. 2020;158:104927.

44. Mehta N, Kalra A, Nowacki AS, Anjewierden S, Han Z, Bhat P, et al. Association of use of angiotensin-converting enzyme inhibitors and angiotensin II receptor blockers with testing positive for coronavirus disease 2019 (COVID-19). JAMA Cardiol. 2020;5:1020-1026.

45. Kai H, Kai M. Interactions of coronaviruses with ACE2, angiotensin II, and RAS inhibitors-lessons from available evidence and insights into COVID-19. Hypertens Res. 2020;43:648-654.

46. Rico-Mesa JS, White A, Anderson AS. Outcomes in patients with COVID-19 infection taking ACEl/ARB. Curr Cardiol Rep. 2020;22:31.

47. Verdecchia $P$, Cavallini $C$, Spanevello A, Angeli F. The pivotal link between ACE2 deficiency and SARS-CoV-2 infection. Eur J Intern Med. 2020;76:14-20. 